\title{
Low Claudin-1 Expression Has Association with Deep Invading and Higher-Grade Colorectal Adenocarcinoma
}

\author{
Putu Erika Paskarani ${ }^{1}$, Herman Saputra ${ }^{1}$, Ni Putu Sriwidyani ${ }^{1}$, I Gusti Ayu Sri Mahendra Dewi ${ }^{1}$, \\ Luh Putu lin Indrayani Maker ${ }^{1}$, I Made Muliartha ${ }^{2}$ \\ ${ }^{1}$ Department of Anatomical Pathology, Medical Faculty of Udayana University, Sanglah General Hospital Denpasar-Bali. \\ ${ }^{2}$ Department of Physiology, Medical Faculty of Udayana University, Bali.
}

\section{ARTICLE INFO}

Received : 09 September 2019

Reviewed : 08 October 2019

Accepted : 11 November 2019

Keywords:

claudin-1, colorectal adenocarcinoma, prognostic factor
ABSTRACT

\begin{abstract}
Background: Claudin-1 is a tight junction protein in the cell that organizes paracellular permeability and epithelial polarity and maintains apical cell-to-cell adhesion. Deregulation of claudin-1 homeostasis will play pivotal role in tumorigenesis, migration, and metastasis of colorectal cancer through a complex signaling pathway. This study investigated the association of claudin-1 expression and clinicopathological factors in colorectal adenocarcinoma.
\end{abstract}

\begin{abstract}
Methods: This study was a cross-sectional study of 43 colorectal cancer patients. Each clinicopathological parameter data was divided into 2 categories; depth of tumor invasion (T3, T4), degree of tumor differentiation (low, high grade), tumor location (right, left), sex (man, woman) and age $(<60, \geq 60$ y.o). Claudin-1 expression was examined by the immunohistochemistry method and evaluated by $\mathrm{H}$-Score method. The $\mathrm{H}$-score cut off was determined by the mean value of 148 . The difference of claudin-1 expression score with tumor depth invasion and age was analyzed by Independent t-test, and then the degree of differentiation, tumor location and sex were analyzed by Mann-Whitney. All variables were analyzed by the Chi-square analysis with a significance value of $P<.05$.
\end{abstract}

Results: There was a significant mean difference of claudin- 1 expression between T3 and T4 tumors ( $P=.041 ; 95 \% \mathrm{Cl} 1.7-75.1$ ). Tumor with low expression of claudin-1 was 2.5 times more likely to be found in tumor with invasion depth T4 rather than T3 ( $P=.023 ; 95 \% \mathrm{Cl} 1.5-48)$. In addition, tumor with low expression of claudin- 1 was 0.2 times more likely to be found in the high-grade tumor ( $P=.001 ; 95 \% \mathrm{Cl} 0.07-0.4)$. Claudin-1 expression was not statistically significant in different tumor locations, sex and age group.

Conclusions: There was mean difference in claudin- 1 expression between T3 and T4 tumors, and between low- and high-grade tumors. The low claudin-1 expression is associated with tumor depth invasion and poorly differentiated tumors. Therefore, the use of claudin- 1 as an additional prognostic factor in colorectal cancer is proposed.

\section{INTRODUCTION}

Claudin-1 is one of the integral membrane proteins that constitute tight junction [1]. It is essential for the tight sealing of the cellular sheet and maintaining homeostasis. Claudin-1 helps to regulate epithelial homeostasis, cellular polarity and barrier dynamics [2]. Carcinogen exposure will disrupt the structure and its function and later plays a pivotal role in tumorigenesis, migration, and metastasis through a complex signaling pathway $[1,3,4]$.

Deregulation of claudin-1 protein in cancer causes loss of cell-to-cell adhesion and it will disrupt its functional signaling pathway. Complex signaling plays a key role in tumorigenesis by either activating or inhibiting cellular processes such as proliferation, differentiation, and apoptosis. Notch and $W n t / \beta$ catenin are one of the important pathways for intestinal maintaining homeostasis [2]. In colorectal cancer, the lack of coordination between Notch and Wnt signaling was shown to be involved in enhancing tumorigenesis $[5,6]$. Crosstalk between it and other signals also induces the epithelial-mesenchymal transition (EMT) process and enhances the metastatic potential of the cancer cells. Researchers suggested that the decrease of claudin-1 expression leads to repression of tight junction and this repression culminates in cell proliferation, motility, and invasion [5-7].

Claudin-1 expression in colorectal cancer has been studied. Kaf et al. [8] showed that claudin-1 expression was found to be significantly related to histological 
grade, morphology subtype, tumor invasion depth, and distant metastasis. This finding is in line with those of Ersoz et al. [3] and Suren et al. [9] On the contrary, Yoshida et al. [10] and Hirakawa et al. [11] found that there was no significant relationship of claudin-1 expression in higher grade tumor and tumor invasion depth. Meanwhile, based on the epidemiological study at Sanglah General Hospital Denpasar in 2014-2016, there were 75 cases $(7.11 \%)$ of rectal cancer and 38 cases (3.60\%) of colorectal cancer [12]. Claudin-1 expression related to adenocarcinoma colorectal cancer had not been studied yet in Bali.

For that reason, our study purposed to determine low claudin-1 expressions related to higher grade and deep invading tumor. The use of claudin-1 as an additional prognostic factor in colorectal cancer is therefore proposed.

\section{METHODS}

\section{Patient selection}

Our study included 55 cases of histopathologically diagnosed colorectal cancer from tissue samples acquired by surgical resection in Sanglah Hospital between in 2017-2019. We excluded 12 patients with incomplete clinicopathological data, treated by neoadjuvant chemotherapy, inappropriate paraffin block. The remaining 43 samples were histopathological re-staged according to the TNM Classification of malignant tumors, 8th Edition of the Union for International Cancer Control (UICC) [13]. We excluded 5 cases of early invasion (T1, T2) of tumor depth due to the small number of samples compared with the late invasion (T3, T4). As a result, only 38 cases of T3, T4 invasion depth were enrolled for analysis. Ethical approval and research permission were obtained from the hospital ethics committee of the Medical Faculty of Udayana University with registration number 1835/UN14.2.2.VII.14/LP/2019. Clinical data such as age, sex, tumor location and history of treatment were obtained by retrospectively searching on computerized hospital database and clinical information data obtained in the histopathological form.

\section{Immunohistochemical staining and evaluation}

Claudin-1 expression was examined by the immunohistochemical method in 43 patients diagnosed with colorectal cancer. Prediluted rabbit polyclonal antibody anti-claudin-1 ready to use, BZ-0883810AF-AP, from bioenzy was used as the primary antibody. Immunohistochemical evaluation was performed by semiquantitative h-score measurement [14]. Both staining intensity (scale $0-3$ ) and the percentage of positive cells (0-100\%) were multiplied to generate an $\mathrm{H}$-score ranging from $0-300$ [14]. The mean $\mathrm{H}$-score was selected as the cut-off value to divide patients into low and high claudin-1 expression. Cases with claudin-1 with a score of $\leq 148$ are classified as low expression and claudin-1 score $>148$ classified as high expression. Normal colon mucosa is a positive membrane staining control [9].

Claudin-1 was evaluated by 2 pathologists (HS, NPS) blinded to patient clinical features. In each case, normal colorectal mucosa adjacent to the carcinoma with complete membranous staining was used as positive internal control. Vascular endothelial and smooth muscle cells, fibroblasts, inflammatory cells, neural and adipocytes were used as the negative internal control [9]. Both staining intensity (scale 0-3) and the percentage of positive cells $(0-100 \%)$ were multiplied to generate an $\mathrm{H}$-score ranging from $\mathrm{O}-300$. $\mathrm{H}$-score numerical data were distributed normally with a mean of 148 . The sample group with $\mathrm{H}$-score less than 148 group has low claudin-1 expression and group with $\mathrm{H}$-score more than or equal with 148 has high claudin-1 expression (Figure 1).

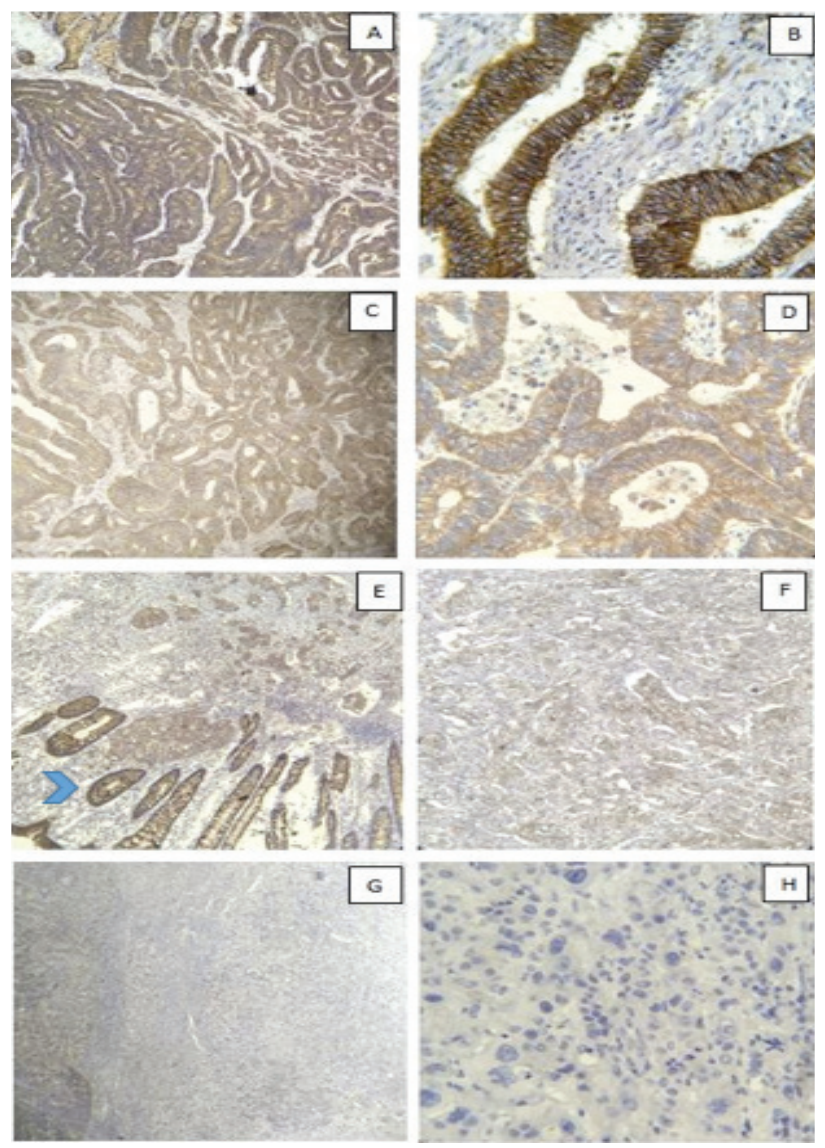

Figure 1. Claudin-1 immunohistochemistry staining. High expression of claudin-1 (A-D); Low expression of claudin-1 (E-H); Nonneoplastic epithelium surface of normal colorectal mucosa as a positive internal control (head arrow, E) 


\section{Statistical Analysis}

Statistical analysis was carried out with IBM SPSS 25 software for Windows 10.0. Data distribution analyzed with Shapiro-Wilk test and p-value above 0.05 are considered as data with normal distribution. Mean $\mathrm{H}$-score data with the depth of tumor invasion T3, T4 and age showed normal data distribution and continued with Independent Sample T-test analysis. Meanwhile, Mean H-score data with tumor location, sex, and age showed abnormal data distribution, which was then analyzed using Mann-Whitney. Furthermore, all variables were enrolled in Chi-Square. $P$-value under 0.05 is significant.

\section{RESULTS}

A total of 43 colorectal cancer patients consisting of $24(55.8 \%)$ males and 19 (44.2\%) females were evaluated. 25 patients (58.1\%) are under 60 years old and 18 (41.9\%) patients above 60 years old. The youngest age is 31 years and the oldest 77 years. All samples were conventional adenocarcinoma whose tumors are located more commonly at the right colon $23(53.5 \%)$ and the rest at left colon 20 (46.5\%). Tumor with depth invasion T1 and T2 was detected in 5 patients and the remaining 38 patients with T3 and T4. Due to the small number of $\mathrm{T} 1$ and $\mathrm{T} 2$, the data were not eligible for further evaluation. As a result, the sample of 38 cases of T3 and T4 tumors were analyzed, consisting of $28(73.7 \%)$ T3 tumors and 10 (26.3\%) T4 tumors. In addition, there were 12 (27.9\%) high-grade tumors and $31(72.1 \%)$ low-grade tumors. Low claudin-1 expression was found in 31 cases (72\%) and high expression in 12 samples (28\%) (Table 1 ).

The Independent T-test analysis revealed there was a mean difference between h-score of claudin-1 expression and tumor invasion depth $(\mathrm{T} 3, \mathrm{~T} 4)(\mathrm{p}=0.041$; 95\% Cl 1.7-75.1) (Table 2). There was no mean difference between $\mathrm{H}$-score of claudin-1 expression and age ( $p=0.256$; 95\% Cl 45.3-11.0) (Table 3).

Mann-Whitney test showed there was a median difference between $\mathrm{H}$-score of claudin-1 expression and degree of differentiation $(p=0.001)$. Meanwhile, there was no median difference between $\mathrm{H}$-score of claudin-1 expression and tumor location $(p=0.954)$ and sex $(p=0.119)$.

In addition, there was a significant difference of claudin-1 expression with tumor invasion depth $(\mathrm{T} 3, \mathrm{~T} 4)$ $(1,5-48,95 \% \mathrm{Cl}, \mathrm{p}=0.023)$ and the degree of tumor differentiation (0.07-0.4, 95\% Cl, p=0.001). Meanwhile, there was no significant difference in claudin-1 expression with tumor location, sex, and age (Table 4).

The analysis was continued to determine the tumor parameter on claudin-1 expression and the result showed only tumor depth invasion and degree of differentiation influence claudin-1 expression $(p=0.023$ and 0.001).

Table 1. Sample characteristics

\begin{tabular}{lcc}
\hline Sample characteristic & Frequency & Percentage \\
\hline Claudin 1 Expression & 31 & $72.1 \%$ \\
Low & 12 & $27.9 \%$ \\
High & & \\
Depth invasion (T) & \\
T3 & 28 & $73.7 \%$ \\
T4 & 10 & $26.3 \%$ \\
Degree of differentiation & & \\
Low & 31 & $72.1 \%$ \\
High & 12 & $27.9 \%$ \\
Location & & \\
Right & 23 & $53.5 \%$ \\
Left & 20 & $46.5 \%$ \\
Sex & & \\
Man & 24 & $55.8 \%$ \\
Woman & 19 & $44.2 \%$ \\
Age (years old) & & \\
$\leq 60$ & 25 & $58.1 \%$ \\
$>60$ & 18 & $41.9 \%$ \\
\hline
\end{tabular}

*Due to small sample of case T1 and T2 (total 5 cases), the data was not eligible for further evaluation

Table 2. H-Score claudin-1 expression and tumor invasion depth $\mathrm{T} 3$ and T4

\begin{tabular}{lcccc}
\hline & $\begin{array}{c}\text { H-score } \\
\text { Mean } \pm \text { SD }\end{array}$ & $\begin{array}{c}\text { Mean } \\
\text { Difference }\end{array}$ & 95\% Cl & P* \\
\hline Invasion depth & & & & \\
T3 $(n=28)$ & $158.93 \pm 43,8$ & 38.43 & $1.7-75.1$ & $0.041^{*}$ \\
T4 $(n=10)$ & $116.7 \pm 62.5$ & & & \\
\hline
\end{tabular}

*Statistically significant $P<.05$

Table 3. H-score claudin-1 expression and age

\begin{tabular}{lcccc}
\hline & $\begin{array}{c}\text { H-score } \\
\text { Mean } \pm \text { SD }\end{array}$ & $\begin{array}{c}\text { Mean } \\
\text { Difference }\end{array}$ & 95\% Cl & P* \\
\hline Age & & & & \\
$\leq 60(n=25)$ & $141.2 \pm 54.8$ & & & \\
$>60(n=18)$ & $158.3 \pm 36.4$ & 17.13 & $47.1-12.9$ & 0.256 \\
\hline
\end{tabular}


Table 4. Tumor parameters of patient adenocarcinoma colorectal based on claudin-1 expression

\begin{tabular}{|c|c|c|c|c|c|c|}
\hline \multirow{2}{*}{ Variable } & \multicolumn{2}{|c|}{ Claudin-1 expression } & \multirow{2}{*}{ Total } & \multirow{2}{*}{$\begin{array}{l}\text { Prevalence } \\
\text { ratio }\end{array}$} & \multirow{2}{*}{$95 \% \mathrm{Cl}$} & \multirow{2}{*}{$\mathbf{p}^{*}$} \\
\hline & low & high & & & & \\
\hline \multicolumn{7}{|c|}{ Depth invasion ( $\mathrm{T}$ ) } \\
\hline T4 & $8(80 \%)$ & $2(20 \%)$ & 10 & \multirow{2}{*}{2.5} & \multirow{2}{*}{$1.5-48$} & \multirow{2}{*}{$0.023^{*}$} \\
\hline T3 & $9(32.1 \%)$ & 19 (67.9\%) & 28 & & & \\
\hline \multicolumn{7}{|c|}{ Degree of differentiation } \\
\hline Low & $5(16 \%)$ & $26(84 \%)$ & 31 & \multirow{2}{*}{0.2} & \multirow{2}{*}{$0.07-0.4$} & \multirow{2}{*}{$0.001^{*}$} \\
\hline High & 12 (100\%) & $0(0 \%)$ & 12 & & & \\
\hline \multicolumn{7}{|c|}{ Tumor location } \\
\hline Left & $8(40 \%)$ & $12(60 \%)$ & 20 & \multirow{2}{*}{0.63} & \multirow{2}{*}{$0.3-3.5$} & \multirow{2}{*}{0.954} \\
\hline Right & 9 (39\%) & $14(61 \%)$ & 23 & & & \\
\hline \multicolumn{7}{|l|}{ Sex } \\
\hline Woman & $5(26 \%)$ & $14(74 \%)$ & 19 & \multirow{2}{*}{0.52} & \multirow{2}{*}{$0.1-1.3$} & \multirow{2}{*}{0.115} \\
\hline Man & $12(50 \%)$ & $12(50 \%)$ & 24 & & & \\
\hline \multicolumn{7}{|l|}{ Age } \\
\hline$\geq 60$ th & $6(33 \%)$ & $12(67 \%)$ & 18 & \multirow{2}{*}{0.75} & \multirow{2}{*}{$0.2-2.2$} & \multirow{2}{*}{0.480} \\
\hline$<60$ th & $11(44 \%)$ & $14(56 \%)$ & 25 & & & \\
\hline
\end{tabular}

*Statistically significant $P<.05$

\section{DISCUSSION}

Previous claudin-1 studies indicate that the loss of claudin-1 function is influenced by changes in APC-beta catenin pathway which is part of the classical pathway $[7,17]$. The loss of claudin-1 function will induce inflammation, epithelial-mesenchymal transition (EMT) and cancer progression $[4,5]$. In addition, the disruption of APC-beta catenin pathway will also induce signal disturbance through a complexity of NOTCH, PI3-K/Akt with RAS-RAF-ERK pathway, loss of apoptotic inhibition, and loss of growth factor, triggering tumorigenesis, migration, and metastasis of tumor $[1,5,6]$.

There is an association between low claudin-1 expression and higher degree of tumor differentiation due to disruption of WNT and Notch signaling pathway. Physiologically, differentiation of the colonic epithelium is tightly regulated by molecular cues in the colonic crypts [2]. These cues are predominately from the WNT and Notch pathway. Notch signaling will determine the cell fate between secretory and absorptive cell lineage [2]. Claudin-1 disruption on cell membrane due to mutation of APC/beta-catenin pathway in adenocarcinoma colorectal will induce the loss of claudin-1 function to keep its cell-to-cell polarity and induce Wnt and Notch signaling disturbance [1]. The loss of cell polarity marked by changes of tumor glandular components which tend to poorly differentiate becomes solid or single cells tumor. By immunohistochemistry staining of claudin-1 protein, the loss of differentiation ability is characterized by the low claudin-1 expression on high grade or poorly differentiated tumor cells (Figure E-F). This study result shows there is a significant difference in h-score claudin-1 expression between low- and high-grade tumors. Low claudin-1 expression was 0.2 times more frequently found in higher grade colorectal adenocarcinoma.

On the other hand, claudin-1 also promotes the translocation of membranous beta-catenin to the nucleus to enhance and activate their proliferation transcription gene, leading to the robust growth of cancer cells or tumorigenesis $[1,5,6]$.

Furthermore, the deregulation of claudin-1 signaling pathway also induces epithelial-mesenchymal transition (EMT) through c-Abl-ERK signaling pathway [5]. It means the cells lose their epithelial characteristics and capability of cell to cell adhesion and gain migratory and invasive properties to become mesenchymal stem cells. These are multipotent stromal cells that can differentiate, invade at the deeper location ( $\mathrm{T}$ ) and initiate metastasis in cancer progression. In addition, other studies also revealed a novel link between claudin-1 and src protein involved in the regulation of apoptosis resistance (anoikis) in colon cancer through claudin-1/Src/PI3k-Akt/ $\mathrm{Bcl}-2$ dependent signaling [5]. Our study revealed low claudin-1 expression was in T3 and T4 tumor depth. Claudin-1 expression was 2.5 more frequently found at T4 to T3 tumor depth. This study also demonstrated tumor invasion depth as an independent factor for claudin-1 expression in colorectal adenocarcinoma. These findings are in line with the theory that " $T$ " is one of the important prognostic factors, especially in colorectal cancer [17].

Another previous study also reported similar findings of the loss of claudin-1 expression related to invasion tumor depth and poorly differentiated colorectal 
adenocarcinoma $[8,10,17]$. In addition, decreased claudin-1 expression is also related to lymph node metastasis and aggressive behavior [3,9]. Another study found reduced expression of claudin-1 is correlated significantly with poor prognosis in stages II and III of rectal cancer [18]. It was found that well- to moderatelydifferentiated adenocarcinoma had significantly more claudin-1 expression than poorly-differentiated adenocarcinoma. Claudin-1 expression decreased in the invasive front of gastric cancer and was negative in many cases with advanced tumor invasion (T2, T3, T4) $[10,18]$.

Our study also analyzes tumor location, sex, and age with a claudin-1 expression that is not statistically significant. Neither do some similar studies find any significant difference in claudin-1 and sex, age and tumor location $[3,10]$. This is in contrast with Huo et al. who found the tumor location is statistically significant with claudin-1 expression. it possibly relates to microsatellite instability which is a part of the colorectal carcinogenesis pathway [18]. Based on the theory, the tumor located at the right colon has the characteristic of high levels of microsatellite instability and the tumor located at the left colon is related to microsatellite-stable without CPG-island methylation $[17,18]$. However, this study had not evaluated it yet and future studies are highly recommended for this carcinogenesis pathway related to tumor location in colorectal cancer. We do not evaluate the disease progression and survival related to claudin-1 expression and colorectal adenocarcinoma yet. Thus, multicenter and prospective studies are required.

In light of all these findings, the claudin-1 protein was the first study in Bali which evaluates its expression, especially in adenocarcinoma colorectal cancer. Our study revealed specifically that low claudin-1 expression was more frequently found in tumor depth invasion T4 than T3. In addition, it also showed that low expression of claudin-1 was more commonly found in a higher degree than a low degree of tumor differentiation. By these findings, the researcher realizes an important perspective of claudin-1 as a tight junction in maintaining cell polarity and its expression related to colorectal adenocarcinoma.

\section{CONCLUSIONS}

Claudin-1 protein expression may become a potential prognostic marker in colorectal cancer. Low expression of claudin-1 expression was found significantly associated with deep tumor invasion and higher-grade tumors. The use of claudin-1 as the additional prognostic factor in colorectal cancer is therefore proposed.

\section{DECLARATIONS}

\section{Competing of Interest}

The authors declare no competing interest in this study.

\section{Acknowledgement}

The authors wish to thank Head of Laboratory Anatomical Pathology Sanglah Hospital Denpasar, mentors and all staff that have contributed, so this research has been done.

\section{REFERENCES}

1. Wang X, Tully O, Ngo B, Zitin M, Mullin J.M. Epithelial tight junctional changes in colorectal cancer tissue. The scientific world journal. 2011;11:826-41.

2. Pope, J.L, Bhat, A.A, Sharma, A, Ahmad, R, Krishnan, $\mathrm{M}$, Washington, M.K, et al. Claudin-1 regulates intestinal epithelial homeostasis through the modulation of Notch-signalling. Br Med J. 2013;63(4):15-6.

3. Ersoz S, Mungan S, Cabanoglu U, Turgutalp H, dan Ozoran Y. Prognostic importance of Claudin-1 and Claudin-4 expression in colon carcinoma. Pathol Res Pract. 2011;97:285-9.

4. Leech A.O, Cruz R.G, Hill A.D, Hopkins A.M. Paradigm lost-an emerging role for over-expression of tight junction adesion proteins in cancer in cancer pathogenesis. Ann Trans Med. 2015;3(13):1-15.

5. Bhat A.A, Uppada S, Achkar I.W, Hashem S, Yadav S.K, Shanmugakonar M, et al. Tight junction protein and signaling pathway in cancer and inflammation: A functional crosstalk. Front Physiol. 2019;9:1-19.

6. Singh A.B, Uppada S.B, Dhawan P. Claudin proteins, outside-in signaling, and carcinogenesis. Eur J Physiol. 2017;469(1):69-75.

7. Stebbing J, Filipovic A, Giamas G. 2013. Claudin-1 as promoter of EMT in hepatocellular carcinoma. Oncogene. 2013;32:4871-72.

8. Kaf R, Saba E, Refaat W, Bahgat A. The Significance of Claudin-1 and S100A4 Expression in Primary Colorectal Carcinoma. Zagazig University Medical Journal. 2016;22:1-12.

9. Suren D, Yildirim M, Kaya V, Alikanoglu A.S, Bulbuller $\mathrm{N}$, Yildiz $\mathrm{M}$, et al. Loss of tight junction proteins (claudin-1,4, and 7) correlates with aggressive behavior in colorectal carcinoma. Med Sci Monit. 2014;1055-62.

10. Yoshida T, Kinugasa T, Akagi Y, Kawahara A, Romeo $K$, Siratsuchi I, et al. Decreased expression of claudin-1 in rectal cancer: A factor for reccurence and poor prognosis. Anticancer Res. 2011;31:2517-26. 
11. Hirakawa E, Tokuhara $Y$, Morinishi T, Ohsaki H, Uemura E, Miki Y, et al. Down regulation of claudin-1 expression in gastric cancer mucosa is correlated with poor prognosis. Open journal of pathology. 2014;4:206-12.

12. Anthonysamy MA, Maker LP, Gotra IM, Saputra H. Prevalence of colorectal carcinoma based on microscopic type, sex, age and anatomic location in sanglah hospital. Intisari Sains Medis. 2018;9(2):119.

13. Brierly JD, James DB, Gospodarowicz MK, Wittekind C. TNM Classification of malignant tumours, 8th Edition. Geneva: Union for International Cancer Control. 2017:84-87

14. Blanchard AA, Ma X, Dueck KJ, Penner C, Cooper SC, Mulhall D, et al. Claudin-1 expression in basallike breast cancer is related to patient age. BMC Cancer. 2013;13(268):1-13.

15. Nagtegaal ID, Arends MJ, Salto-Tellez M. Colorectal adenocarcinoma. In: Lokuhetty D., White VA,
Watanabe R, dan Cree IA. World Health Organization Classification of Tumours, 5th edition. France: International Agency for Research on Cancer. 2018:177-88.

16. Huo Q, Kinugasa $T$, Wang L, Huang J, Zhao J, Shibaguchi $\mathrm{H}$, et al. Claudin-1 protein is a major factor involved in the tumorigenesis of colorectal cancer. Anticancer Res. 2009;29:851-8.

17. Skoufi U, Arvanitis DL, Lampri L, loachim E, Koutsogiannis J, Skoufi C, et al. Association of Claudin-1 with E-Cadherin/Catenin Complex, Microvessel Density (MVD)-Related Markers and Clinicopathological Feature in Colorectal Carcinoma. J Interdiscip Histopathol. 2014;2:135-44.

18. Hamilton SR, Bosman FT, Boffeta P, llyas M, Morreau $\mathrm{H}$. Carcinoma of the colon and rektum. In: Hamilton S.R, Bosman F.T, Carneiro F, Hruban H.R, Theise N.D. World Health Organization Classification of Tumours, 4th edition. France: International Agency for Research on Cancer. 2010:134-5. 